\title{
Numerical ranges of composition operators on $l^{2}$
}

\author{
B.S. Komal and Shallu Sharma
}

(Received September 1, 2003; Revised September 27, 2004)

Abstract. In this paper we obtain numerical ranges of composition operators on $l^{2}$.

Key words: numerical range, composition operator, orbit.

\section{Preliminaries}

Let $\mathbb{N}$ be the set of all positive integers and $l^{2}=l^{2}(\mathbb{N})$ be the Hilbert space of all square summable sequences of complex numbers. Let $T$ be a mapping of $\mathbb{N}$ into itself. Then we can define a composition transformation $C_{T}$ from $l^{2}(\mathbb{N})$ into the space of all complex valued sequences by $C_{T} f=f o T$ for every $f \in l^{2}(\mathbb{N})$. In the case $C_{T}$ is bounded and the range of $C_{T}$ is contained in $l^{2}(\mathbb{N})$, we call it a composition operator induced by $T$. It is shown in [9] that a composition trasformation $C_{T}: l^{2}(\mathbb{N}) \rightarrow l^{2}(\mathbb{N})$ is a bounded operator if and only if there exists a real number $M>0$ such that $\#\left(T^{-1}(\{n\})\right) \leq M$ for every $n \in N$, where $\#(E)$ denotes the cardinality of the set $E$. Thus it is evident that a composition transformation $C_{T}$ is unbounded if and only if the sequence $\left\{\#\left(T^{-1}(\{n\})\right)\right\}_{n=1}^{\infty}$ is an unbounded sequence. From Theorem 3.1 of Singh and Komal [9], it follows that $C_{T}$ is surjective if and only if $T$ is injective.

The numerical range of a bounded linear operator $A: H \rightarrow H$ from Hilbert space $H$ into itself is defined as $W(A)=\{\langle A x, x\rangle:\|x\|=1\}$, where $\langle.,$.$\rangle is the inner product of H$. The numerical radius of $A$ is defined as $w(A)=\sup \{|\langle A x, x\rangle|:\|x\|=1\}$. These concepts and their generalisations have been studied extensively because of their connections and applications to many different branches of Mathematics (e.g. see chapter I of Horn and Johnson [12]).

Let $T: N \rightarrow N$ be a mapping. Two positive integers $m$ and $n$ are said to be in the same orbit of $T$ if there exit two positive integers $r$ and $s$ such that $T^{r}(m)=T^{s}(n)$. Here and else where, $T^{r}$ denotes the composition of $T$ with itself $r$ times. If $n \in N$, then $O_{T}(n)=\left\{m \in N: T^{r}(m)=T^{s}(n)\right.$ for

2000 Mathematics Subject Classification : Primary 47B99, Secondary 47B38. 
some $r, s \in N\}$ is called the orbit of $n$ with respect to $T$.

A mapping $T: N \rightarrow N$ is said to be antiperiodic at $n$, if $T^{m}(n) \neq n$ for every $m \in N$. If $T$ is antiperiodic at every $n \in N$, then we say that $T$ is purely antiperiodic. For an example, the mapping $T: N \rightarrow N$ defined by

$$
T(n)=\left\{\begin{array}{cc}
n+2, & \text { if } n \text { is even } \\
n, & \text { if } n \text { is odd }
\end{array}\right.
$$

is antiperiodic at every even natural number but not antiperiodic at an odd natural number. If for a natural number $n$ there exists $m \in N$ such that $T^{m}(n)=n$, then $T$ is called periodic at $n$. If $T$ is periodic at every $n \in N$, we say that $T$ is purely periodic. The integer $m_{n}=\inf \left\{m: T^{m}(n)=n\right\}$ is called the period of $T$ at $n$. The set $\left\{m_{n}: n \in N\right\}$ of periods of $T$ is denoted by $P(T)$. For sake of convenience, we shall use $f_{0}(n)$ to denote the cardinality of the set $T^{-1}(\{n\})$. The smallest convex set containing the set $G \subset l^{2}(\mathbb{N})$ is called the convex hull of $G$ and we shall denote it by $C_{o}(G)$. The spectrum of a bounded operator $A$ on a Hilbert space $H$ into itself is defined by $\sigma(A)=\{\lambda \in \mathbb{C}: A-\lambda I$ is not invertible $\}$. If $F \subset N$, then $\chi_{F}$ denotes the characteristics function of $F$. It is well known that $\left\{\chi_{\{n\}}: n \in N\right\}$ is an orthonormal basis for $l^{2}(\mathbb{N})$. The Banach algebra of all bounded linear operators from $l^{2}(\mathbb{N})$ into itself is denoted by $B\left(l^{2}(\mathbb{N})\right)$. For $E \subset N, l^{2}(E)=\left\{f \in l^{2}(\mathbb{N}): f(m)=0\right.$ for every $\left.m \notin E\right\}$. The symbol $C_{T} \mid l^{2}(E)$ denotes the restriction of $C_{T}$ to $l^{2}(E)$.

The composition operators have been the subject matter of a systematic study over the past three decades (e.g. see monographs Cowen and Maccluer [2], Shapiro [11] and Singh and Manhas [13]). For more information concerning composition operators on $l^{2}(\mathbb{N})$ and numerical ranges of operators, we refer to Carlson [1], Singh and Komal ([9] and [10]), Gustafson and Rao [3], Halmos [4], Matache [7] and Stout [14]. In this paper we compute the numerical ranges of composition operators on $l^{2}(\mathbb{N})$. It is shown that numerical range of an unbounded composition operator is the entire complex plane.

\section{Numerical ranges of surjective composition operators}

In this section we obtain the numerical ranges of surjective composition operators. 
Lemma 2.1 Let $T: N \rightarrow N$ be injective and antiperiodic at some $n \in$ $N$ and let $S$ be the restriction of $T$ to $O_{T}(n)$. Then $W\left(C_{S}\right)=\{\lambda \in \mathbb{C}$ : $|\lambda|<1\}$, where $C_{S}$ is a composition operator on $l^{2}\left(O_{T}(n)\right)$ induced by $S$.

Proof. If $S$ is invertible, then by Theorem 2.3 of Singh and Komal [9], $C_{S}$ is normal and therefore $\overline{W\left(C_{S}\right)}=C_{o}\left(\sigma\left(C_{S}\right)\right)$ in view of theorm 1.4.5 of Gustafason and Rao [3]. Since $\sigma\left(C_{S}\right)=\{\lambda:|\lambda|=1\}$, it follows that $\overline{W\left(C_{S}\right)}=\{\lambda:|\lambda| \leq 1\}$. If $|\lambda|=1$ and $\lambda \in W\left(C_{S}\right)$, then $|\lambda|=\left|\left\langle C_{s} f, f\right\rangle\right| \leq$ $\left\|C_{S} f\right\|\|f\| \leq 1$ for some unit vector $f \in l^{2}\left(O_{T}(n)\right)$ implies that Schwartz inequality becomes an equality. Hence $C_{S} f$ and $f$ are linearly dependent so that $C_{S} f=\mu f$ for some scalar $\mu$. This contradicts the fact that $C_{S}$ has no eigenvalue in the light of Lemma 2.1 of Komal and Pathania [6]. Thus $W\left(C_{S}\right) \subset\{\lambda \in \mathbb{C}:|\lambda|<1\}$. To prove the reverse inclusion, let $\lambda=r e^{i \theta}$, where $0<r<1,0 \leq \theta \leq 2 \pi$. For $O_{T}(n)=\left\{n_{k}\right\}_{k=1}^{\infty}$ where $S\left(n_{k}\right)=n_{k+1}$, define $f=\sum_{k=1}^{\infty} \sqrt{\left(1-r^{2}\right)} r^{k-1} e^{-i(k-1) \theta} \chi_{\left\{n_{k}\right\}}$. A simple computation reveals that $\|f\|=1$ and $\left\langle C_{S} f, f\right\rangle=r e^{i \theta}$. This proves that $\{\lambda:|\lambda|<1\} \subset W\left(C_{s}\right)$. Hence $W\left(C_{S}\right)=\{\lambda:|\lambda|<1\}$. Next, if $S$ is not invertible in $O_{T}(n)$, then $S$ is not surjective and hence $S^{-1}\left(\left\{n_{1}\right\}\right)=\phi$. Taking $f$ as defined above, we see that $\left\langle C_{S} f, f\right\rangle=r e^{i \theta}, 0<r<1,0 \leq$ $\theta \leq 2 \pi$. This proves that $\{\lambda \in \mathbb{C}:|\lambda|<1\} \subseteq W\left(C_{S}\right)$. Now $S$ is injective. Therefore by corollary to Theorem 2.1.9 of Singh and Komal [9], $\left\|C_{s}\right\|=1$. Hence $W\left(C_{S}\right) \subseteq\{\lambda \in \mathbb{C}:|\lambda| \leq 1\}$. As proved earlier, if $|\lambda|=1$, then $\lambda \notin W\left(C_{S}\right)$. Thus in this case also $W\left(C_{S}\right)=\{\lambda:|\lambda|<1\}$.

Lemma 2.2 Let $T: N \rightarrow N$ be an injection and periodic at some $n \in N$. Then

(i) $W\left(C_{S}\right)=\{1\}$, if $\#\left(O_{T}(n)\right)=1$,

(ii) $W\left(C_{S}\right)=[-1,1]$, if $\#\left(O_{T}(n)\right)=2$,

(iii) $W\left(C_{S}\right)$ is the closed polygonal region whose boundary is the regular polygon with \# $\left(O_{T}(n)\right)$ sides inscribed in the unit circle having one of the vertex at 1 , where $S$ is the restriction of $T$ to $O_{T}(n)$.

Proof. If $\#\left(O_{T}(n)\right)=1$, then $S$ is the identity map and so trivially $W\left(C_{S}\right)=\{1\}$. Further if $\#\left(O_{T}(n)\right) \geq 2$, then $S$ is periodic of period $k=\#\left(O_{T}(n)\right)$. Clearly $S$ is invertible. This implies that $C_{S}$ is invertible and hence $C_{S}$ is normal by Theorem 2.3 of Singh and Komal [9]. From corollary to Theorem 3.3 of singh and Komal [10], it follows that $\sigma\left(C_{S}\right)=$ $\left\{\lambda \in \mathbb{C}: \lambda^{k}=1\right\}$. By using normality of $C_{S}$, and the fact that range of an 
operator on a finite dimensional space is always closed, we have $W\left(C_{S}\right)=$ $\overline{W\left(C_{S}\right)}=C_{o}\left(\sigma\left(C_{S}\right)\right)$. We have thus proved that $W\left(C_{S}\right)=C_{o}\{\lambda \in \mathbb{C}:$ $\left.\lambda^{k}=1\right\}$. In particular, for $k=2, W\left(C_{S}\right)=[-1,1]$.

Theorem 2.3 Let $T: N \rightarrow N$ be injective. Then

(i) $W\left(C_{T}\right)=\{\lambda \in \mathbb{C}:|\lambda|<1\}$, if $T$ is purely antiperiodic

(ii) $W\left(C_{T}\right)=C_{o}\left(\bigcup_{n \in P(T)}\left\{\lambda \in \mathbb{C}: \lambda^{n}=1\right\}\right)$, if $T$ is purely periodic.

(iii) $W\left(C_{T}\right)=C_{o}\left(\{\lambda \in \mathbb{C}:|\lambda|<1\} \cup \bigcup_{k \in P(T)}\left\{\lambda \in \mathbb{C}: \lambda^{k}=1\right\}\right)$, if $T$ is periodic at some $n \in N$ and antiperiodic at some other point $m \in N$.

Proof. The proof of (i) follows from Lemma 2.1. For the proof of (ii), write $l^{2}(\mathbb{N})=\sum_{n \in P(T)} \oplus l^{2}\left(O_{T}(n)\right)$ so that

$$
\begin{aligned}
W\left(C_{T}\right) & =C_{o}\left(\bigcup_{n \in P(T)} W\left(C_{T} \mid l^{2}\left(O_{T}(n)\right)\right)\right) \\
& =C_{o}\left(\bigcup_{n \in P(T)}\left\{\lambda \in \mathbb{C}: \lambda^{n}=1\right\}\right),
\end{aligned}
$$

where $C_{T} \mid l^{2}\left(O_{T}(n)\right)$ is restriction of $C_{T}$ to $l^{2}\left(O_{T}(n)\right)$. For the proof of (iii), let $E=\{n \in N: T$ is periodic at $n\}$. Then $l^{2}(\mathbb{N})=l^{2}(E) \oplus l^{2}(N-E)$. Hence that

$$
\begin{aligned}
W\left(C_{T}\right) & =C_{o}\left(W\left(C_{T} \mid l^{2}(E)\right) \mathrm{U} W\left(C_{T} \mid l^{2}(N-E)\right)\right) \\
& =C_{o}\left(\bigcup_{k \in P(T)}\left\{\lambda \in \mathbb{C}: \lambda^{k}=1\right\} \mathrm{U}\{\lambda \in \mathbb{C}:|\lambda|<1\}\right)
\end{aligned}
$$

follows by using the above parts (i) and (ii).

\section{Numerical Ranges of Non-Surjective Composition Operators}

In this section we obtain the numerical ranges of non-surjective composition operators.

Lemma 3.1 Let $C_{T} \in B\left(l^{2}(\mathbb{N})\right)$. Then $W\left(C_{T} / l^{2}\left(E_{n}\right)\right)=\{\lambda \in \mathbb{C}:|\lambda| \leq$ $\left.\sqrt{f_{0}(n)} / 2\right\}$, where $n$ is such that $f_{0}(n) \geq 2, T(n) \neq n ; T^{2}(n) \neq n$ and $E_{n}=T^{-1}(\{n\}) \mathrm{U}\{n\}$. 
Proof. Suppose $f_{0}(n)=k \geq 2$ and $E_{n}=\left\{n, n_{1} n_{2} \ldots n_{k}\right\}$. Clearly

$$
f=f_{n} \chi_{\{n\}}+f_{n_{1}} \chi_{\left\{n_{1}\right\}}+\cdots+f_{n_{k}} \chi_{\left\{n_{k}\right\}} \in l^{2}\left(E_{n}\right),
$$

and

$$
\left\langle C_{T} f, f\right\rangle=\sum_{i=1}^{k} f_{n} \overline{f_{n_{i}}} .
$$

Therefore $\left|\left\langle C_{T} f, f\right\rangle\right| \leq\left|f_{n}\right| \sum_{i=1}^{k}\left|f_{n_{i}}\right|$. In order to calculate the numerical radius of $C_{T} \mid l^{2}\left(E_{n}\right)$, we compute $\sup \left\{\left|f_{n}\right| \sum_{i=1}^{k}\left|f_{n_{i}}\right|\right\} \ldots$ (1) subject to the condition $\left|f_{n}\right|^{2}+\sum_{i=1}^{k}\left|f_{n_{i}}\right|^{2}=1$. Let $r_{i}=\left|f_{n_{i}}\right|$ for $i=1,2, \ldots, k$ and $r_{n}=\left|f_{n}\right|$. Consider the Lagranges function $F\left(r_{1}, r_{2} \ldots r_{k}, r_{n}, \lambda\right)=$ $r_{n} \sum_{i=1}^{k} r_{i}-\lambda\left(\sum_{i=1}^{k} r_{i}^{2}+r_{n}^{2}-1\right)$. Therefore, solving the equations $\partial F / \partial r_{n}=$ $\sum_{i=1}^{k} r_{i}-2 \lambda r_{n}=0, \partial F / \partial r_{i}=r_{n}-2 \lambda r_{i}=0$, for $i=1,2, \ldots, k$, the maximum expression (1) is $\sqrt{f_{0}(n)} / 2$. By the definition the numerical radius of $C_{T}$ restricted to $l^{2}\left(E_{n}\right)$ is the maximum expression (1). Therefore $w\left(C_{T} \mid l^{2}\left(E_{n}\right)\right)=\sqrt{f_{0}(n)} / 2$. This proves that $W\left(C_{T} \mid l^{2}\left(E_{n}\right)\right) \subset\{\lambda:|\lambda| \leq$ $\left.\sqrt{f_{0}(n)} / 2\right\}$. Taking $f=\cos \alpha \chi_{\{n\}}+(\sin \alpha / \sqrt{k}) e^{-i \theta} \chi_{\left\{n_{1}, n_{2} \ldots n_{k}\right\}}$, we get $\|f\|=1$ and

$$
\left\langle C_{T} f, f\right\rangle=\frac{k \cos \alpha \sin \alpha e^{i \theta}}{\sqrt{k}}=\frac{\sqrt{k}}{2} \sin 2 \alpha e^{i \theta}=r e^{i \theta},
$$

where $r=(\sqrt{k} / 2) \sin 2 \alpha \leq \sqrt{k} / 2$. This proves that $\{\lambda \in \mathbb{C}:|\lambda| \leq$ $\left.\sqrt{f_{0}(n)} / 2\right\} \subset W\left(C_{T}\right)$. Thus $W\left(C_{T} \mid l^{2}\left(E_{n}\right)\right)=\left\{\lambda \in \mathbb{C}:|\lambda| \leq \sqrt{f_{0}(n)} / 2\right\}$.

Lemma 3.2 Let $C_{T} \in B\left(l^{2}(N)\right)$. Then $W\left(C_{T} \mid l^{2}\left(E_{n}\right)\right) \subseteq\{\lambda \in \mathbb{C}:|\lambda| \leq$ $\left.\sqrt{f_{0}(n)+3} / 2\right\}$, where $n$ is such that $f_{0}(n) \geq 2, T(n) \neq n$ but $T^{2}(n)=n$, and $E_{n}=T^{-1}(\{n\}) \cup\{n\}$.

Proof. Suppose $f_{0}(n)=k \geq 2$ and $E_{n}=\left\{n, n_{1} n_{2} \ldots n_{k}\right\}$. From the hypothesis, $T(n)=n_{j}$ for some $j, 1 \leq j \leq k$. Clearly $f=f_{n} \chi_{\{n\}}+f_{n_{1}} \chi_{\left\{n_{1}\right\}}+$ $\cdots+f_{n_{k}} \chi_{\left\{n_{k}\right\}} \in l^{2}\left(E_{n}\right)$, and $\left|\left\langle C_{T} f, f\right\rangle\right| \leq\left|f_{n}\right| \sum_{i=1}^{k}\left|f_{n_{i}}\right|+\left|f_{n}\right|\left|f n_{j}\right|$. We shall now compute $\sup \left\{\left|f_{n}\right| \sum_{i=1}^{k}\left|f_{n_{i}}\right|+\left|f_{n}\right|\left|f_{n_{j}}\right|\right\} \ldots$. (1) subject to the condition $\sum_{i=1}^{k}\left|f_{n_{i}}\right|^{2}+\left|f_{n}\right|^{2}=1$. For this, consider the Lagrange's function $F\left(r_{1}, r_{2} \ldots, r_{n}, \lambda\right)=r_{n} \sum_{i=1}^{k} r_{i}+r_{n} r_{j}-\lambda\left(\sum_{i=1}^{k} r_{i}^{2}+r_{n}^{2}-1\right)$, where $r_{i}=\left|f_{n_{i}}\right|$ and $r_{n}=\left|f_{n}\right|$. Solving the equations $\partial F / \partial r_{n}=\sum_{i=1}^{k} r_{i}+r_{j}-2 \lambda r_{n}=0$, $\partial F / \partial r_{j}=2 r_{n}-2 \lambda r_{j}=0$, and $\partial F / \partial r_{i}=r_{n}-2 \lambda r_{i}=0$, for $i=1,2, \ldots, k$, 
$i \neq j$, we find that the maximum expression (1) is equal to $\sqrt{f_{0}(n)+3} / 2$. This gives $w\left(C_{T} \mid l^{2}\left(E_{n}\right)\right)=\sqrt{f_{0}(n)+3} / 2$ which proves the lemma.

Lemma 3.3 Let $C_{T} \in B\left(l^{2}(\mathbb{N})\right)$. Then $W\left(C_{T} \mid l^{2}\left(E_{n}\right)\right)$ is the closed elliptical disc with foci at 0 and 1 and major axis $=\sqrt{f_{0}(n)}$ and minor axis $=\sqrt{f_{0}(n)-1}$, where $f_{0}(n) \geq 2$ and $T(n)=n, E_{n}=T^{-1}\{n\}$.

Proof. Let $f_{0}(n)=k \geq 2$ and $E_{n}=T^{-1}(\{n\})=\left\{n, n_{1}, \ldots, n_{k-1}\right\}$. Then the range of $C_{T} \mid l^{2}\left(E_{n}\right)$ is the one dimensional subspace of $l^{2}(N)$ spanned by $\chi_{E_{n}}$. For $h=\chi_{\left\{n, n_{1}, \ldots, n_{k-1}\right\}} / \sqrt{k}$, and $g=\sqrt{k} \chi_{\{n\}},\left(C_{T} \mid l^{2}\left(E_{n}\right)\right)(f)=$ $\langle f, g\rangle h$ for every $f \in l^{2}\left(E_{n}\right)$.

Also $\|h\|=1$. Hence by the two dimensional Case 2.4 and Proposition 2.5 of Bourdon and Shapiro [5], $W\left(C_{T} \mid l^{2}\left(E_{n}\right)\right)$ is a closed elliptical disc with foci at 0 and 1 , major axis $=\sqrt{f_{0}(n)}$ and minor axis $=\sqrt{f_{0}(n)-1}$. This proves the lemma.

Theorem 3.4 Let $C_{T} \in B\left(l^{2}(N)\right)$ be such that $T$ is purely antiperiodic but not injective. Then $W\left(C_{T}\right) \supseteq\left\{\lambda:|\lambda| \leq\left\|\sqrt{f_{0}}\right\|_{\infty} / 2\right\}$, where $\left\|f_{0}\right\|_{\infty}=$ $\sup \left\{f_{0}(n): n \in N\right\}$.

Proof. Let $n \in N$ be such that $f_{0}(n)=\sup \left\{f_{0}(m): m \in N\right\}=\left\|f_{0}\right\|_{\infty}$. This is possible, since by Theorem 2.1 of Singh and Komal [9] range of $f_{0}$ is a finite set. By using Lemma 3.1 for any $m \in N$, we obtain $W\left(C_{T} \mid l^{2}\left(E_{m}\right)\right)=$ $\left\{\lambda \in \mathbb{C}:|\lambda| \leq \sqrt{f_{0}(m)} / 2\right\}$. In particular for $m=n, \mathrm{~W}\left(C_{T} \mid l^{2}\left(E_{n}\right)\right)=$ $\left\{\lambda \in \mathbb{C}:|\lambda| \leq\left\|\sqrt{f_{0}}\right\|_{\infty} / 2\right\}$. Hence $W\left(C_{T}\right) \supseteq\left\{\lambda \in \mathbb{C}:|\lambda| \leq\left\|\sqrt{f_{0}}\right\|_{\infty} / 2\right\}$.

Theorem 3.5 Let $T: N \rightarrow N$ be a mapping such that $C_{T}: D\left(\subseteq l^{2}(N)\right) \rightarrow$ $l^{2}(N)$ is an unbounded operator, where $D$, the domain of $C_{T}$ is a dense subspace of $l^{2}(\mathbb{N})$. Then $W\left(C_{T}\right)=\mathbb{C}$, the complex plane.

Proof. Suppose $C_{T}$ is an unbounded operator. Then by Theorem 2.1 of Singh and Komal [9], there exists an increasing sequence $\left\{n_{k}\right\}$ of positive integers such that $f_{0}\left(n_{k}\right) \rightarrow \infty$ as $k \rightarrow \infty$. Now two cases can arise:

Case 1: $T\left(n_{k}\right) \neq n_{k}$ for infinitely many values of $k$. In this case either $T^{2}\left(n_{k}\right) \neq n_{k}$ for infinitely many values of $k$ or $T^{2}\left(n_{k}\right)=n_{k}$ for all but finitely many values of $k$. Now if $T^{2}\left(n_{k}\right) \neq n_{k}$ for infinitely many values of $k$, then by Lemma 3.1, $W\left(C_{T} \mid l^{2}\left(E_{n_{k}}\right)\right)=\left\{\lambda \in \mathbb{C}:|\lambda| \leq \sqrt{f_{0}\left(n_{k}\right)} / 2\right\}$ for infinitely many values of $k$. But $W\left(C_{T} \mid l^{2}\left(E_{n_{k}}\right)\right) \subset W\left(C_{T}\right)$ for every $k$. Therefore $\mathbb{C} \subseteq W\left(C_{T}\right)$. Next suppose $T^{2}\left(n_{k}\right)=n_{k}$ for all but finitely many 
values of $k$. Let $T^{-1}\left\{n_{k}\right\}=\left\{p_{1 k}, p_{2 k} \ldots, p_{m_{k} k}\right\}$ and $T\left(n_{k}\right)=p_{j k}$ for some $j$, $1 \leq j \leq m_{k}$ as $T\left(n_{k}\right) \in T^{-1}\left(\left\{n_{k}\right\}\right)$. Now $f_{0}\left(n_{k}\right)=m_{k} \rightarrow \infty$ as $k \rightarrow \infty$. Take

$$
\begin{aligned}
f= & \frac{1}{\sqrt{2}} \chi_{\left\{n_{k}\right\}}+\frac{e^{i \theta}}{\sqrt{2\left(m_{k}+3\right)}} \chi_{\left\{p_{1 k}, \ldots p_{(j-1) k}, p_{(j+1) k} \ldots p_{m_{k} k}\right\}} \\
& +\sqrt{\frac{2}{\left(m_{k}+3\right)}} \chi_{\left\{p_{j k}\right\}},
\end{aligned}
$$

for $0 \leq \theta \leq 2 \pi$. A simple computation reveals that $\|f\|=1$ and

$$
\left\langle C_{T} f, f\right\rangle=\frac{m_{k}-1}{\sqrt{2}} \frac{e^{i \theta}}{\sqrt{2\left(m_{k}+3\right)}}+\frac{2}{\sqrt{m_{k}+3}} .
$$

Therefore, $2 / \sqrt{m_{k}+3}+\left(m_{k}-1\right) /\left(2 \sqrt{m_{k}+3}\right) e^{i \theta} \in W\left(C_{T}\right)$ for $0 \leq \theta \leq 2 \pi$ which implies that

$$
\left\{z \in \mathbb{C}:\left|z-\frac{2}{\sqrt{m_{k}+3}}\right| \leq \frac{m_{k}-1}{2 \sqrt{m_{k}+3}}\right\} \subset W\left(C_{T}\right) \ldots \ldots(*),
$$

since numerical range of an operator is convex. We now show that $\mathbb{C} \subseteq$ $W\left(C_{T}\right)$. Since $m_{k} \rightarrow \infty$, choose $m_{k}$ so large that $\sqrt{m_{k}+3} / 2 \geq|\lambda|+4 \geq$ $|\lambda|+4 / \sqrt{m_{k}+3}$. This implies that

$$
\begin{aligned}
\left|\lambda-\frac{2}{\sqrt{m_{k}+3}}\right| & \leq|\lambda|+\frac{2}{\sqrt{m_{k}+3}} \\
& \leq \frac{\sqrt{m_{k}+3}}{2}-\frac{4}{\sqrt{m_{k}+3}}+\frac{2}{\sqrt{m_{k}+3}} \\
& =\frac{m_{k}+1}{2 \sqrt{m_{k}+3}} .
\end{aligned}
$$

Hence by $\left(^{*}\right) \lambda \in W\left(C_{T}\right)$.

Case II: $T\left(n_{k}\right)=n_{k}$ for all but finitely many values of $k$. An application of Lemma 3.3 yields that the closed elliptical disc with foci at 0,1 , major axis $=\sqrt{f_{0}\left(n_{k}\right)}$ and minor axis $=\sqrt{f_{0}\left(n_{k}\right)-1}$ is contained in $W\left(C_{T}\right)$. Since $f_{0}\left(n_{k}\right) \rightarrow \infty$, we conclude that $\mathbb{C} \subseteq W\left(C_{T}\right)$. This completes the proof of the theorem.

We shall now give an example of an unbounded composition operator.

Example 3.6 Let $D=\left\{f \in l^{2}(\mathbb{N}): f(n)=0\right.$ for all but finitely many values of $n\}$. Then $D$ is a dense linear subspace of $l^{2}(\mathbb{N})$. Let $T: N \rightarrow N$ 
be defined as

$$
T(p)=\left\{\begin{array}{cc}
2^{2^{m}}+1, & \text { if } p \in\left[2^{2^{m-1}}+1,2^{2^{m}}\right] \text { for } m=1,2, \ldots \\
3, & \text { if } p \in\{1,2\}
\end{array}\right.
$$

where $[m, n]$ denotes the set of all positive integers $p$ such that $m \leq p \leq n$. Then $f_{0}(p)=\#\left(T^{-1}(\{p\})\right) \rightarrow \infty$ as $p \rightarrow \infty$. Therefore $C_{T}: D \rightarrow l^{2}(N)$ is not bounded.

Theorem 3.7 Let $C_{T} \in B\left(l^{2}(N)\right)$ and $T$ be such that $T^{2}(n)=T(n)$ for every $n \in N$. Then

(i) $W\left(C_{T}\right)=\{1\}$, if $T$ is the identity map.

(ii) $W\left(C_{T}\right)=C_{0}\left(\cup_{n \in T(N)} D n\right)$, if $T$ is not the identity map, where Dn is the elliptical disc with foci at 0,1 , major axis $=\sqrt{f_{0}(n)}$ and minor axis $=\sqrt{f_{0}(n)-1}$.

Proof. The proof of part (i) is trivial. For the proof of part (ii) if $n \in T(N)$, write $E_{n}=T^{-1}(\{n\})$. Now $C_{T}\left(l^{2}\left(E_{n}\right)\right) \subset l^{2}\left(E_{n}\right)$ and $C_{T}\left(l^{2}\left(N-E_{n}\right)\right) \subseteq$ $l^{2}\left(N-E_{n}\right)$. Therefore, $C_{T}=\sum_{n \in T(N)} \oplus\left(C_{T} \mid l^{2}\left(E_{n}\right)\right)$ because $l^{2}(N)=$ $\sum_{n \in T(N)} \oplus l^{2}\left(E_{n}\right)$. Hence $W\left(C_{T}\right)=C_{0}\left(\bigcup_{n \in T(N)}^{\infty} W\left(C_{T} \mid l^{2}\left(E_{n}\right)\right)\right)$. But by Lemma 3.3, $W\left(C_{T} \mid l^{2}\left(E_{n}\right)\right)=D_{n}$. Therefore the proof of the theorem is complete.

In [5] Bourdon and Shapiro considered the zero inclusion question i.e. for which $T$ does $\mathrm{W}\left(C_{T}\right)$ contain the origin? This is proved in Theorem 3.1 of [5] that if $T$ is not identity, $0 \in \overline{W\left(C_{T}\right)}$. We in the following theorem show that $W\left(C_{T}\right)$ always contains the origin when $T \neq I$.

Theorem 3.8 Let $C_{T} \in B\left(l^{2}(N)\right)$ and $T$ be not the identity map. Then $0 \in W\left(C_{T}\right)$.

Proof. If $T$ is not surjective, then we can choose a positive integer $n_{0} \notin$ $T(N)$. Clearly $\left\|\chi_{\left\{n_{0}\right\}}\right\|=1$ and $\left\langle C_{T} \chi_{\left\{n_{0}\right\}}, \chi_{\left\{n_{0}\right\}}\right\rangle=o$. Thus $0 \in W\left(C_{T}\right)$. Next, suppose $T$ is surjective. Now if $T$ is injective, then since $T \neq I$, so there exists $n \in N$ for which $T(n) \neq n$. Again $\left\langle C_{T} \chi_{\{n\}}, \chi_{\{n\}}\right\rangle=o$. Further, if $T$ is not injective, then $T\left(n_{1}\right)=T\left(n_{2}\right)=n$ (say) for two distict positive integers $n_{1}$ and $n_{2}$. Atleast one of $n_{1}$ and $n_{2}$, say $n_{1}$ is distinct from $n$. By surjectivity of $T, T\left(n_{0}\right)=n_{1}$ for some $n_{0} \in N$. Moreover, $n_{1} \notin T^{-1}\left(\left\{n_{1}\right\}\right)$; otherwise $T\left(n_{1}\right)=n_{1}$ and so $n_{1}=n$ which contradicts the choice of $n$. Clearly $\left\langle C_{T} \chi_{\left\{n_{1}\right\}}, \chi_{\left\{n_{1}\right\}}\right\rangle=0$. Thus $0 \in W\left(C_{T}\right)$. 


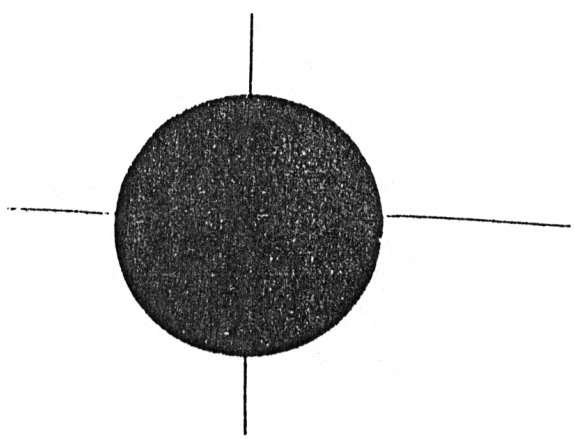

Fig. 3.1.

Example 3.9 Let $T: N \rightarrow N$ be defined by $T(n)=p n+q, p, q \in N$. Then $W\left(C_{T}\right)=\{\lambda:|\lambda|<1\}$.

Example 3.10 Let $T: N \rightarrow N$ be defined by $T(n)=\sum_{j=0}^{k+1} 2^{2 j}$ if $n \in$ $\left[\sum_{j=0}^{k} 2^{2 j}, \sum_{j=1}^{k+1} 2^{2 j}\right]$ for $k=0,1,2,3, \ldots$ Then $C_{T}: D \rightarrow l^{2}(N)$ is an unbounded operator where $D$ is given in Example 3.6. Then

$$
r_{k}=\frac{\sqrt{f_{0}\left(\sum_{j=0}^{k+1} 2^{2 j}\right)}}{2}=\frac{\sqrt{2^{2(k+1)}}}{2}=\frac{2^{(k+1)}}{2} .
$$

Then in view of Lemma 3.1 the closed $\operatorname{disc}\left\{\lambda \in \mathbb{C}:|\lambda| \leq 2^{k}\right\} \subset W\left(C_{T}\right)$ for each $k=0,1,2, \ldots$ It follows that $\mathbb{C} \subset W\left(C_{T}\right)$. Hence $W\left(C_{T}\right)=\mathbb{C}$.

Example 3.11 Let $T: N \rightarrow N$ be defined by

$$
T(n)=\left\{\begin{array}{cl}
1, & \text { if } n \in\{1,2\} \\
1+\sum_{j=0}^{k} 2^{2^{j}}, & \text { if } n \in\left[1+\sum_{j=0}^{k} 2^{2^{j}}, \sum_{j=0}^{k+1} 2^{2^{j}}\right] \\
& \text { for } k=0,1,2,3,4,5 \\
n, & \text { if } n>\sum_{j=0}^{6} 2^{2^{j}}
\end{array}\right.
$$

Then $f_{0}(1)=2, f_{0}\left(1+\sum_{j=0}^{k} 2^{2^{j}}\right)=2^{2^{k+1}}$ for $k=0,1,2, \ldots, 5$. By Lemma 3.3, $W\left(C_{T}\right)=D_{1} \cup \bigcup_{i=0}^{5} D_{m_{k}}$, where $D_{m_{k}}$ is an elliptical disc with foci at 0,1 , major axis $=\sqrt{2}$ and minor axis $=1, D_{m_{k}}$ is an elliptical disc with foci at 0,1 , major axis $=2^{2^{k}}$ and minor axis $=\sqrt{2^{2 k+1}-1}$, for 


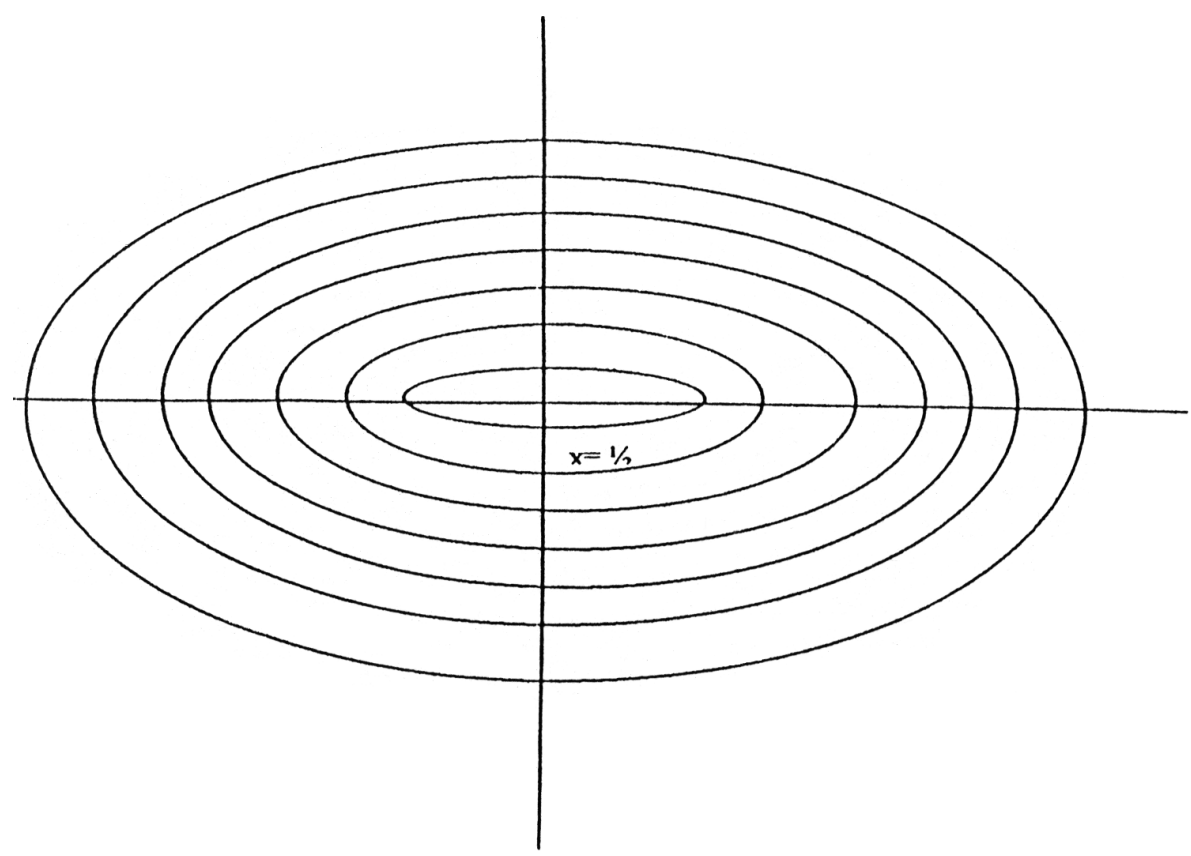

Fig. 3.2.

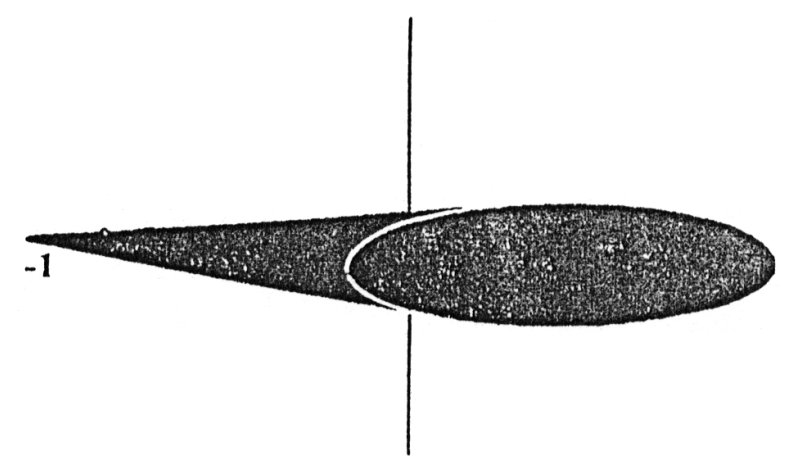

Fig. 3.3.

$k=0,1,2,3,4,5$ and $m_{k}=1+\sum_{j=0}^{k} 2^{2^{j}}$.

\section{Example 3.12 (i)}

$$
T(n)= \begin{cases}n+1, & \text { if } n \text { is odd } \\ n-1, & \text { if } n \text { is even }\end{cases}
$$


(ii)

$$
T(n)=\left\{\begin{array}{cl}
n+1, & \text { if } n=1 \\
n-1, & \text { if } n=2 \\
n, & \text { if } n \text { is odd } \geq 3 \\
n-1, & \text { if } n \text { is even } \geq 3
\end{array}\right.
$$

$W\left(C_{T}\right)=C_{0}\left(\{-1\} U D_{1}\right)$, where $D_{1}$ is ellipse with foci at 0,1 and major axis $=\sqrt{2}$ and minor axis $=1$.

\section{References}

[1] Carlson J.W., The spectra and commutant of some weighted composition operators, Trans. Amer. Math. Soc., 317 (1990), 631-654.

[2] Cowen C.C. and Maccluer B.D., Composition operators on spaces of analytic functions, CRC Press Boca Raton, 1995.

[3] Gustafson K.E. and Rao D.K.M., Numerical Range, The field of values of Linear operators and Matrices, Springer Verlag, New York, 1997.

[4] Halmos P.R. Hilbert space Problem Book, Van Nostrand, Princeton, New Jersey, 1997.

[5] Bourdon P.S. and Shapiro H., When is zero in the numerical range of a composition operator?, Integ. Equ. Oper. Theory, 44 (2002), 410-441.

[6] Komal B.S. and Pathania R.S., On Eigenvalues of composition operators on $l^{2}$, Indian J. Pure and Appl. Math., 22 (3), 213-219.

[7] Matache V., Numerical ranges of composition operators, Linear Algebra and its application, 331 (2001), 61-74.

[8] Ridge W.C., Numerical Ranges of a weighted shift with periodic weights, Proc. Amer. Math. Soc., 55 (1976), 107-110.

[9] Singh R.K. and Komal B.S., Composition operators on $l^{p}$ and its adjoint, Proc. Amer. Math. Soc., 70 (1978), 21-25.

[10] Singh R.K. and Komal B.S., Composition operators, Bull. Austral. Math. Soc., 18 (1970), 439-446.

[11] Shapiro J.H., Composition operators and classical Function theory, Springer Verlag, 1993.

[12] Horn R.A. and Johnson C.R., Topics in matrix anaysis, Cambridge University press, New York, 1991.

[13] Singh R.K. and Manhas J.S., Composition operators on function spaces, NorthHolland Mathematics Studies 179, Elsevier Science Publishers Amsterdam, New York, 1993.

[14] Stout Q.F., The Numerical range of a weighted shift, Proc. Amer. Math. Soc., 88 (1983), 495-502. 
B.S. Komal

Department of Mathematics,

University of Jammu,

Jammu- 180006. (INDIA)

E-mail: bskomal2@yahoo.co.in

S. Sharma

Department of Mathematics,

University of Jammu,

Jammu- 180006. (INDIA)

E-mail: shalluk3@yahoo.co.in 